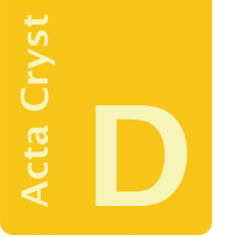

STRUCTURAL BIOLOGY

Volume 72 (2016)

Supporting information for article:

Crystal structure of the human interferon gamma receptor 2 reveals the structural basis for receptor specificity

Pavel Mikulecký, Jirí Zahradník, Petr Kolenko, Jirí Cerný, Tatsiana Charnavets, Lucie Kolárová, Iva Necasová, Phuong Ngoc Pham and Bohdan Schneider 


\section{S1. Methods}

S1.1. Cloning, expression, and purification of endoglycosidases. Endoglycosidases Endo $\mathrm{H}$ (residues 42-313; UniProt P04067) and PNGase F (residues 8-299; UniProt Q9XBM8; (Cancino-Diaz et al., 2002)) were produced in Escherichia coli BL21 ( $\lambda$ DE3) and purified before deglycosylation trials. Briefly, genes were obtained as a GeneArt Strings DNA Fragments and cloned into previously modified vector pET26b $(+)$ bearing StrepTag instead of 6x HisTag using NdeI and XhoI restriction enzymes. Cells were grown in $1 \mathrm{~L}$ of LB medium at $30{ }^{\circ} \mathrm{C}$ until the $\mathrm{OD}_{600}=0.6$ was reached, then the temperature was decreased to $16{ }^{\circ} \mathrm{C}$ and protein expression was induced by addition of $1 \mathrm{mM}$ IPTG, and cells were incubated for another 20 hours. Two-step purification of enzymes consisted of gravity flow StrepTactin Sepharose followed by size exclusion chromatography on a HiLoad 16/600 Superdex 75 pg (GE Healthcare).

S1.2. Thermal shift assay (TSA). Melting temperature curves was also obtained from fluorescence-based Thermal shift assay using fluoroprobe. Experiment was performed in "CFX96 Touch Real-Time PCR Detection System" (Bio-Rad) using FRET Scan Mode. The concentration of fluorescent SYPRO Orange dye (Sigma Aldrich) was 8-fold dilution from 5,000 -fold stock and protein concentration was $0.05 \mathrm{mg} / \mathrm{mL}$ in final volume of $25 \mu \mathrm{L}$. As a reference was used only "HN Buffer" without protein. Thermal denaturation of proteins was performed in capped "Low Tube Strips, CLR" (Bio-Rad) and possible air bubbles in samples were removed by centrifugation immediately before the assay. The samples were heated from 20 to $95{ }^{\circ} \mathrm{C}$ with stepwise increment of $0.5^{\circ} \mathrm{C} / \mathrm{min}$ and a $30 \mathrm{~s}$ hold step for every point, followed by the fluorescence reading. Data (after subtraction of reference sample) were normalized and used for first derivative calculation to estimate the melting temperature. 
Table S1. Primers for mutagenesis. The IFNgR2 variants bearing single mutations: N110Q, N137Q, and N231Q were produced using the QuikChange II Site-Directed Mutagenesis Kit (Agilent Technologies). Primer pair (forward primer - Fwd and reverse - Rev) are marked by mutation code.

\begin{tabular}{|c|c|}
\hline FwdN137Q: & 5 '-GTTTCAACACTATCGGCAAGTGACTGTCGGGCCTC-3' \\
\hline RevN137Q: & $5^{\prime}$-GAGGCCCGACAGTCACTTGCCGATAGTGTTGAAAC-3' \\
\hline FwdN110Q: & 5 ' -CTTCCCAATGGATTTCCAGGTCACTCTACGCCTTCG-3' \\
\hline RevN1100: & 5 ' -CGAAGGCGTAGAGTGACCTGGAAATCCATTGGGAAG-3 ' \\
\hline FwdN231Q: & $5^{\prime}$ '-GAGTCGGGCATTTAAGCCAGATATCTTGCTACGAAACAATG-3' \\
\hline RevN2310: & 5 '-CATTGTTTCGTAGCAAGATATCTGGCTTAAATGCCCGACTC-3' \\
\hline
\end{tabular}

Table S3. The mutual orientation of the D1 and D2 domains of the cytokine receptors measured as torsion angles. The torsion angles are defined by $\mathrm{CA}$ atoms of four residues highly conserved among all receptors.

\begin{tabular}{ccccccc}
$\begin{array}{c}\text { PDB and } \\
\text { chain IDs }\end{array}$ & $\begin{array}{c}\text { Receptor } \\
\text { name }\end{array}$ & Torsion & \multicolumn{3}{c}{ Residues defining torsion between D1 and D2 } \\
\hline 5eh1_A & IFNgR2 & $-38^{\circ}$ & A92 & R89 & Y150 & V152 \\
3lqm_A & IL10R2 & $-49^{\circ}$ & A91 & R88 & Y154 & V156 \\
1fg9_C & IFNgR1 & $-30^{\circ}$ & A87 & R84 & Y155 & V157 \\
1j7v_R & IL10R1 & $-14^{\circ}$ & A81 & R78 & Y146 & I148 \\
4doh_B & IL20R2 & $-82^{\circ}$ & A115 & R112 & F165 & V167 \\
4doh_E & IL20R1 & $-27^{\circ}$ & A114 & R111 & Y181 & V183 \\
3dlq_R & IL22R & $-21^{\circ}$ & A98 & R95 & Y165 & L167 \\
3g9v_A & IL22BP & $-40^{\circ}$ & A105 & R102 & Y171 & V173 \\
30g6_B & IL28R & $-17^{\circ}$ & T82 & R79 & Y138 & V140 \\
\hline
\end{tabular}


Table S2. Amino acid residues (numbered as in the PDB structures) used to calculate root mean square deviations (rmsd, [Å]) for the D1 and D2 domains of the twelve analyzed receptor molecules listed in Figure 3. Note that the number of superimposed residues is the same in all domains, 34.

Calculation of rmsd of 34 residues of the $\mathrm{N}$-terminal D1 domain

\begin{tabular}{|c|c|c|c|c|c|c|c|c|c|c|c|c|c|c|c|c|c|c|c|c|c|c|c|c|c|c|c|c|c|c|c|c|c|c|c|c|}
\hline $\begin{array}{l}\text { 음 } \\
\text { 믐 }\end{array}$ & 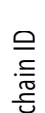 & $\begin{array}{l}\overline{\bar{a}} \\
\overline{0} \\
\text { dूँ }\end{array}$ & & & & & & & & & & & & & & res & . & 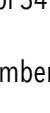 & as nu & iberec & in th & PDB & ile & & & & & & & & & & & & & \\
\hline $1 \mathrm{fg} 9$ & $C$ & IFNgR1 & 28 & 29 & 30 & 31 & 41 & 42 & 43 & 44 & 45 & 46 & 47 & 56 & 57 & 58 & 59 & 60 & 65 & 66 & 67 & 68 & 69 & 81 & 82 & 83 & 84 & 85 & 86 & 87 & 88 & 94 & 96 & 97 & 98 & 99 \\
\hline 5eh1 & A & IFNgR2 & 21 & 22 & 23 & 24 & 37 & 38 & 39 & 40 & 41 & 42 & 43 & 49 & 50 & 51 & 52 & 61 & 66 & 67 & 68 & 69 & 70 & 86 & 87 & 88 & 89 & 90 & 91 & 92 & 93 & 99 & 101 & 102 & 103 & 104 \\
\hline $1 \mathrm{j} 7 \mathrm{v}$ & $\mathrm{R}$ & IL10R1 & 21 & 22 & 23 & 24 & 35 & 36 & 37 & 38 & 39 & 40 & 41 & 48 & 49 & 50 & 51 & 52 & 57 & 58 & 59 & 60 & 61 & 75 & 76 & 77 & 78 & 79 & 80 & 81 & 82 & 88 & 90 & 91 & 92 & 93 \\
\hline $31 \mathrm{qm}$ & A & IL10R2 & 37 & 38 & 39 & 40 & 51 & 52 & 53 & 54 & 55 & 56 & 57 & 62 & 63 & 64 & 65 & 66 & 71 & 72 & 73 & 74 & 75 & 85 & 86 & 87 & 88 & 89 & 90 & 91 & 92 & 98 & 100 & 101 & 102 & 103 \\
\hline 4doh & B & IL20R2 & 51 & 52 & 53 & 54 & 65 & 66 & 67 & 68 & 69 & 70 & 71 & 83 & 84 & 85 & 86 & 89 & 94 & 95 & 96 & 97 & 98 & 109 & 110 & 111 & 112 & 113 & 114 & 115 & 116 & 122 & 124 & 125 & 126 & 127 \\
\hline 4doh & $E$ & IL20R1 & 53 & 54 & 55 & 56 & 68 & 69 & 70 & 71 & 72 & 73 & 74 & 81 & 82 & 83 & 84 & 87 & 92 & 93 & 94 & 95 & 96 & 108 & 109 & 110 & 111 & 112 & 113 & 114 & 115 & 121 & 123 & 124 & 125 & 126 \\
\hline $3 d \mathrm{dq}$ & $\mathrm{R}$ & IL22R & 38 & 39 & 40 & 41 & 52 & 53 & 54 & 55 & 56 & 57 & 58 & 65 & 66 & 67 & 68 & 71 & 76 & 77 & 78 & 79 & 80 & 92 & 93 & 94 & 95 & 96 & 97 & 98 & 99 & 106 & 107 & 108 & 109 & 110 \\
\hline $3 g 9 v$ & A & IL22BP & 44 & 45 & 46 & 47 & 59 & 60 & 61 & 62 & 63 & 64 & 65 & 72 & 73 & 74 & 75 & 78 & 83 & 84 & 85 & 86 & 87 & 99 & 100 & 101 & 102 & 103 & 104 & 105 & 106 & 112 & 114 & 115 & 116 & 117 \\
\hline 3096 & B & IL28R & 20 & 21 & 22 & 23 & 34 & 35 & 36 & 37 & 38 & 39 & 40 & 48 & 49 & 50 & 51 & 54 & 59 & 60 & 61 & 62 & 63 & 76 & 77 & 78 & 79 & 80 & 81 & 82 & 83 & 89 & 91 & 92 & 93 & 94 \\
\hline 3 se4 & A & IFNabR1 & 17 & 18 & 19 & 20 & 33 & 34 & 35 & 36 & 37 & 38 & 39 & 46 & 47 & 48 & 49 & 52 & 57 & 58 & 59 & 60 & 61 & 73 & 74 & 75 & 76 & 77 & 78 & 79 & 80 & 86 & 87 & 88 & 89 & 90 \\
\hline 3 se4 & C & IFNabR2 & 24 & 25 & 26 & 27 & 38 & 39 & 40 & 41 & 42 & 43 & 44 & 52 & 53 & 54 & 55 & 58 & 63 & 64 & 65 & 66 & 67 & 79 & 80 & 81 & 82 & 83 & 84 & 85 & 86 & 93 & 94 & 95 & 96 & 97 \\
\hline $2 p u q$ & T & Tissue_F & 22 & 23 & 24 & 25 & 33 & 34 & 35 & 36 & 37 & 38 & 39 & 45 & 46 & 47 & 48 & 49 & 54 & 55 & 56 & 57 & 58 & 71 & 72 & 73 & 74 & 75 & 76 & 77 & 78 & 93 & 94 & 95 & 96 & 97 \\
\hline
\end{tabular}

Calculation of rmsd of 34 residues of the $\mathrm{C}$-terminal $\mathrm{D} 2$ domain

\begin{tabular}{|c|c|c|c|c|c|c|c|c|c|c|c|c|c|c|c|c|c|c|c|c|c|c|c|c|c|c|c|c|c|c|c|c|c|c|c|c|}
\hline 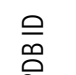 & $\begin{array}{l}\text { 으 } \\
\underset{c}{\mathbb{c}}\end{array}$ & $\begin{array}{l}\overline{\overline{0}} \\
\overline{0}\end{array}$ & & & & & & & & & & & & & & & du & Imb & 2 & $m b$ r & $d$ in $t$ & e PDB f & & & & & & & & & & & & & & \\
\hline $1 \mathrm{fg} 9$ & $C$ & IFNgR1 & 113 & 114 & 115 & 116 & 117 & 118 & 124 & 125 & 126 & 127 & 128 & 129 & 152 & 154 & 155 & 156 & 157 & 158 & 159 & 184 & 185 & 186 & 187 & 197 & 198 & 199 & 200 & 201 & 202 & 203 & 204 & 213 & 214 & 216 \\
\hline 5eh1 & A & IFNgR2 & 118 & 120 & 121 & 122 & 123 & 124 & 130 & 131 & 132 & 133 & 134 & 135 & 148 & 149 & 150 & 151 & 152 & 153 & 154 & 171 & 172 & 173 & 174 & 184 & 185 & 186 & 187 & 188 & 189 & 190 & 191 & 203 & 204 & 206 \\
\hline $1 j 7 v$ & $\mathrm{R}$ & IL10R1 & 106 & 108 & 109 & 110 & 111 & 112 & 118 & 119 & 120 & 121 & 122 & 123 & 144 & 145 & 146 & 147 & 148 & 149 & 150 & 168 & 169 & 170 & 171 & 181 & 182 & 183 & 184 & 185 & 186 & 187 & 188 & 197 & 198 & 200 \\
\hline $31 \mathrm{gm}$ & A & IL10R2 & 116 & 117 & 118 & 119 & 120 & 121 & 127 & 128 & 129 & 130 & 131 & 132 & 152 & 153 & 154 & 155 & 156 & 157 & 158 & 175 & 176 & 177 & 178 & 188 & 189 & 190 & 191 & 192 & 193 & 194 & 195 & 204 & 205 & 207 \\
\hline 4doh & B & IL2OR2 & 141 & 142 & 143 & 144 & 145 & 146 & 152 & 153 & 154 & 155 & 156 & 157 & 163 & 164 & 165 & 166 & 167 & 168 & 169 & 189 & 190 & 191 & 192 & 202 & 203 & 204 & 205 & 206 & 207 & 208 & 209 & 218 & 219 & 221 \\
\hline 4doh & $\mathrm{E}$ & IL20R1 & 140 & 141 & 142 & 143 & 144 & 145 & 151 & 152 & 153 & 154 & 155 & 156 & 179 & 180 & 181 & 182 & 183 & 184 & 185 & 202 & 203 & 204 & 205 & 215 & 216 & 217 & 218 & 219 & 220 & 221 & 222 & 232 & 233 & 234 \\
\hline $3 \mathrm{dlq}$ & $R$ & IL22R & 124 & 125 & 126 & 127 & 128 & 129 & 135 & 136 & 137 & 138 & 139 & 140 & 163 & 164 & 165 & 166 & 167 & 168 & 169 & 185 & 186 & 187 & 188 & 198 & 199 & 200 & 201 & 202 & 203 & 204 & 205 & 214 & 215 & 216 \\
\hline $3 \mathrm{~g} 9 \mathrm{v}$ & A & IL22BP & 131 & 132 & 133 & 134 & 135 & 136 & 142 & 143 & 144 & 145 & 146 & 147 & 169 & 170 & 171 & 172 & 173 & 174 & 175 & 192 & 193 & 194 & 195 & 206 & 207 & 208 & 209 & 210 & 211 & 212 & 213 & 222 & 223 & 225 \\
\hline $30 g 6$ & B & IL28R & 108 & 109 & 110 & 111 & 112 & 113 & 119 & 120 & 121 & 122 & 123 & 124 & 136 & 137 & 138 & 139 & 140 & 141 & 142 & 161 & 162 & 163 & 164 & 175 & 176 & 177 & 178 & 179 & 180 & 181 & 182 & 192 & 193 & 195 \\
\hline 3 se4 & A & IFNabR1 & 104 & 105 & 106 & 107 & 108 & 109 & 115 & 116 & 117 & 118 & 119 & 120 & 136 & 137 & 138 & 139 & 140 & 141 & 142 & 159 & 160 & 161 & 162 & 172 & 173 & 174 & 175 & 176 & 177 & 178 & 179 & 188 & 189 & 191 \\
\hline $3 \mathrm{se} 4$ & C & IFNabR2 & 110 & 111 & 112 & 113 & 114 & 115 & 121 & 122 & 123 & 124 & 125 & 126 & 139 & 140 & 141 & 142 & 143 & 144 & 145 & 167 & 168 & 169 & 170 & 180 & 181 & 182 & 183 & 184 & 185 & 186 & 187 & 195 & 196 & 198 \\
\hline $2 p u q$ & $\mathrm{~T}$ & Tissue_F & 111 & 112 & 113 & 115 & 116 & 117 & 123 & 124 & 125 & 126 & 127 & 128 & 151 & 152 & 153 & 154 & 155 & 156 & 157 & 174 & 175 & 176 & 177 & 186 & 187 & 188 & 189 & 190 & 191 & 192 & 193 & 204 & 205 & 207 \\
\hline
\end{tabular}


residue number as numbered in the PDB file

\begin{tabular}{|c|c|c|c|c|c|c|c|c|c|c|c|c|c|c|c|c|c|c|c|c|c|c|c|c|c|c|c|c|c|c|c|c|c|c|c|c|}
\hline $1 \mathrm{fg} 9$ & $l$ & NgR1N & 15 & 18 & 19 & 20 & 24 & 26 & 27 & 30 & 40 & 42 & 43 & 44 & 45 & 46 & 47 & 48 & 64 & 66 & 67 & 68 & 80 & 81 & 82 & 84 & 35 & 86 & 87 & 88 & 89 & 91 & 2 & 93 & 102 & 03 \\
\hline $1 \mathrm{fg} 9$ & C & IgR1C & 113 & 115 & 116 & 117 & 121 & 23 & 124 & 127 & 152 & 155 & 156 & 157 & 158 & 159 & 160 & 161 & 172 & 183 & 184 & 185 & 195 & 196 & 197 & 199 & 200 & 201 & 202 & 203 & 204 & 208 & 209 & 210 & 219 & 20 \\
\hline 5eh1 & A & VgR2N & 8 & 9 & 10 & 11 & 12 & 13 & 14 & 15 & 21 & 22 & 23 & 24 & 38 & 39 & 40 & 41 & 42 & 45 & 64 & 65 & 66 & 67 & 68 & 69 & 81 & 87 & 88 & 89 & 90 & 91 & 93 & 97 & 101 & 102 \\
\hline eh1 & A & $\operatorname{~IgR2C~}$ & 141 & 142 & 143 & 144 & 145 & 149 & 151 & 152 & 153 & 155 & 168 & 169 & 170 & 171 & 172 & 173 & 174 & 175 & 177 & 178 & 179 & 180 & 181 & 182 & 183 & 184 & 185 & 195 & 196 & 198 & 199 & 200 & 201 & 202 \\
\hline $1 j 7 v$ & $\mathrm{r}$ & 10R1N & 7 & 8 & 9 & 10 & 11 & 13 & 20 & 21 & 24 & 25 & 32 & 39 & 40 & 41 & 45 & 46 & 47 & 50 & 57 & 58 & 59 & 60 & 61 & 62 & 67 & 74 & 76 & 77 & 78 & 79 & 81 & 82 & 89 & 90 \\
\hline $1 j 7 v$ & & 10R1C & 105 & 106 & 107 & 108 & 109 & 111 & 118 & 119 & 122 & 123 & 142 & 149 & 150 & 151 & 156 & 157 & 158 & 161 & 166 & 167 & 168 & 169 & 170 & 171 & 174 & 179 & 181 & 182 & 183 & 184 & 186 & 187 & 196 & 197 \\
\hline $3 \mathrm{lqm}$ & A & L10R2N & 27 & 29 & 37 & 48 & 50 & 51 & 52 & 53 & 54 & 56 & 60 & 63 & 64 & 71 & 72 & 73 & 74 & 75 & 76 & 86 & 87 & 88 & 89 & 90 & 91 & 92 & 93 & 95 & 96 & 103 & 104 & 105 & 106 & 107 \\
\hline $31 \mathrm{qm}$ & $A$ & L10R2C & 118 & 120 & 128 & 149 & 152 & 153 & 154 & 155 & 156 & 158 & 164 & 167 & 168 & 173 & 174 & 175 & 176 & 177 & 178 & 188 & 189 & 190 & 191 & 192 & 193 & 194 & 195 & 199 & 200 & 208 & 209 & 210 & 211 & 212 \\
\hline 4doh & B & IL20R1N & 63 & 66 & 67 & 68 & 69 & 70 & 71 & 72 & 73 & 74 & 75 & 77 & 79 & 88 & 89 & 102 & 104 & 106 & 107 & 108 & 109 & 110 & 111 & 112 & 113 & 114 & 115 & 119 & 121 & 122 & 123 & 124 & 125 & 126 \\
\hline 4doh & B & IL20R1C & 171 & 174 & 176 & 178 & 179 & 180 & 181 & 182 & 183 & 184 & 185 & 186 & 191 & 193 & 194 & 195 & 196 & 197 & 198 & 210 & 213 & 214 & 215 & 216 & 217 & 218 & 219 & 220 & 221 & 222 & 224 & 226 & 234 & 235 \\
\hline 4doh & E & IL20R2N & 38 & 52 & 64 & 65 & 66 & 67 & 68 & 69 & 70 & 71 & 72 & 98 & 99 & 108 & 109 & 110 & 111 & 112 & 113 & 114 & 115 & 116 & 117 & 118 & 119 & 120 & 121 & 122 & 123 & 124 & 125 & 130 & 131 & 132 \\
\hline 4doh & E & IL2OR2C & 141 & 154 & 163 & 164 & 165 & 166 & 167 & 168 & 169 & 170 & 171 & 191 & 192 & 200 & 201 & 202 & 203 & 204 & 205 & 206 & 207 & 208 & 209 & 210 & 213 & 214 & 215 & 216 & 217 & 218 & 219 & 223 & 224 & 225 \\
\hline 3dlq & R & IL22RN & 20 & 22 & 25 & 26 & 27 & 28 & 29 & 30 & 39 & 41 & 43 & 44 & 45 & 46 & 47 & 48 & 51 & 52 & 53 & 54 & 55 & 56 & 57 & 58 & 59 & 62 & 90 & 98 & 100 & 104 & 105 & 106 & 107 & 112 \\
\hline 3dlq & R & IL22RC & 121 & 122 & 123 & 124 & 125 & 126 & 134 & 164 & 165 & 166 & 167 & 168 & 169 & 170 & 171 & 198 & 200 & 202 & 203 & 204 & 210 & 211 & 212 & 213 & 214 & 215 & 216 & 217 & 218 & 219 & 220 & 221 & 222 & 224 \\
\hline $3 g 9 v$ & A & IL22BPN & 30 & 31 & 34 & 35 & 36 & 43 & 44 & 45 & 46 & 48 & 49 & 50 & 58 & 59 & 60 & 61 & 62 & 63 & 64 & 75 & 81 & 82 & 83 & 100 & 101 & 102 & 103 & 104 & 105 & 111 & 112 & 113 & 114 & 115 \\
\hline $3 g 9 v$ & A & IL22BPC & 130 & 131 & 133 & 134 & 135 & 142 & 143 & 144 & 145 & 147 & 148 & 149 & 169 & 170 & 171 & 172 & 173 & 174 & 175 & 186 & 189 & 190 & 191 & 206 & 207 & 208 & 209 & 210 & 211 & 219 & 220 & 221 & 222 & 223 \\
\hline $30 \mathrm{~g} 6$ & B & IL28RN & 7 & 17 & 19 & 20 & 21 & 24 & 31 & 32 & 34 & 35 & 36 & 37 & 38 & 39 & 40 & 46 & 58 & 62 & 63 & 64 & 65 & 74 & 76 & 79 & 80 & 81 & 82 & 83 & 87 & 88 & 89 & 90 & 91 & 92 \\
\hline 3096 & B & IL28RC & 108 & 116 & 119 & 120 & 121 & 124 & 134 & 135 & 137 & 138 & 139 & 140 & 141 & 142 & 143 & 147 & 157 & 162 & 163 & 164 & 165 & 172 & 174 & 177 & 178 & 179 & 180 & 181 & 188 & 189 & 190 & 191 & 192 & 193 \\
\hline 3 se4 & A & IFNabR1N & 11 & 13 & 18 & 19 & 20 & 33 & 34 & 35 & 36 & 37 & 38 & 39 & 40 & 41 & 47 & 48 & 54 & 58 & 59 & 60 & 71 & 72 & 73 & 74 & 75 & 76 & 77 & 78 & 79 & 80 & 87 & 88 & 93 & 94 \\
\hline $3 \mathrm{se} 4$ & A & IFNabR1C & 107 & 109 & 117 & 118 & 119 & 137 & 138 & 139 & 140 & 141 & 142 & 143 & 144 & 145 & 151 & 152 & 155 & 158 & 159 & 160 & 169 & 170 & 171 & 172 & 173 & 174 & 175 & 176 & 177 & 178 & 188 & 189 & 194 & 195 \\
\hline 3 se4 & C & IFNabR2N & 39 & 40 & 41 & 42 & 43 & 50 & 52 & 53 & 54 & 59 & 66 & 67 & 68 & 69 & 70 & 72 & 73 & 74 & 77 & 78 & 79 & 80 & 81 & 82 & 83 & 84 & 85 & 94 & 95 & 96 & 97 & 98 & 99 & 100 \\
\hline 3 se4 & C & IFNabR2C & 141 & 142 & 143 & 144 & 145 & 149 & 151 & 152 & 153 & 155 & 168 & 169 & 170 & 171 & 172 & 173 & 174 & 175 & 177 & 178 & 179 & 180 & 181 & 182 & 183 & 184 & 185 & 195 & 196 & 198 & 199 & 200 & 201 & 202 \\
\hline ouq & $\mathrm{T}$ & ssueFN & 6 & 13 & 14 & 22 & 23 & 24 & 25 & 26 & 35 & 36 & 39 & 40 & 41 & 44 & 45 & 46 & 47 & 54 & 55 & 56 & 57 & 58 & 59 & 70 & 71 & 72 & 73 & 74 & 75 & 76 & 93 & 94 & 99 & 100 \\
\hline $2 p u q$ & $\mathrm{~T}$ & TissueFC & 108 & 115 & 116 & 124 & 125 & 126 & 127 & 128 & 154 & 155 & 158 & 159 & 160 & 164 & 165 & 166 & 167 & 172 & 173 & 174 & 175 & 176 & 177 & 184 & 185 & 186 & 187 & 188 & 189 & 190 & 203 & 204 & 209 & 210 \\
\hline
\end{tabular}


Figure S1. SDS-PAGE analysis of the purified glycosylated and deglycosylated IFNgR2. The $12.5 \%$ Tris-Glycine gel was stained using Coomassie Brilliant Blue. Lane $\mathrm{M}$ - molecular-weight markers with depicted mass in $\mathrm{kDa}$; Lane R2g glycosylated IFNgR2; Lane R2d - IFNgR2 deglycosylated by Endo H endoglycosidase.

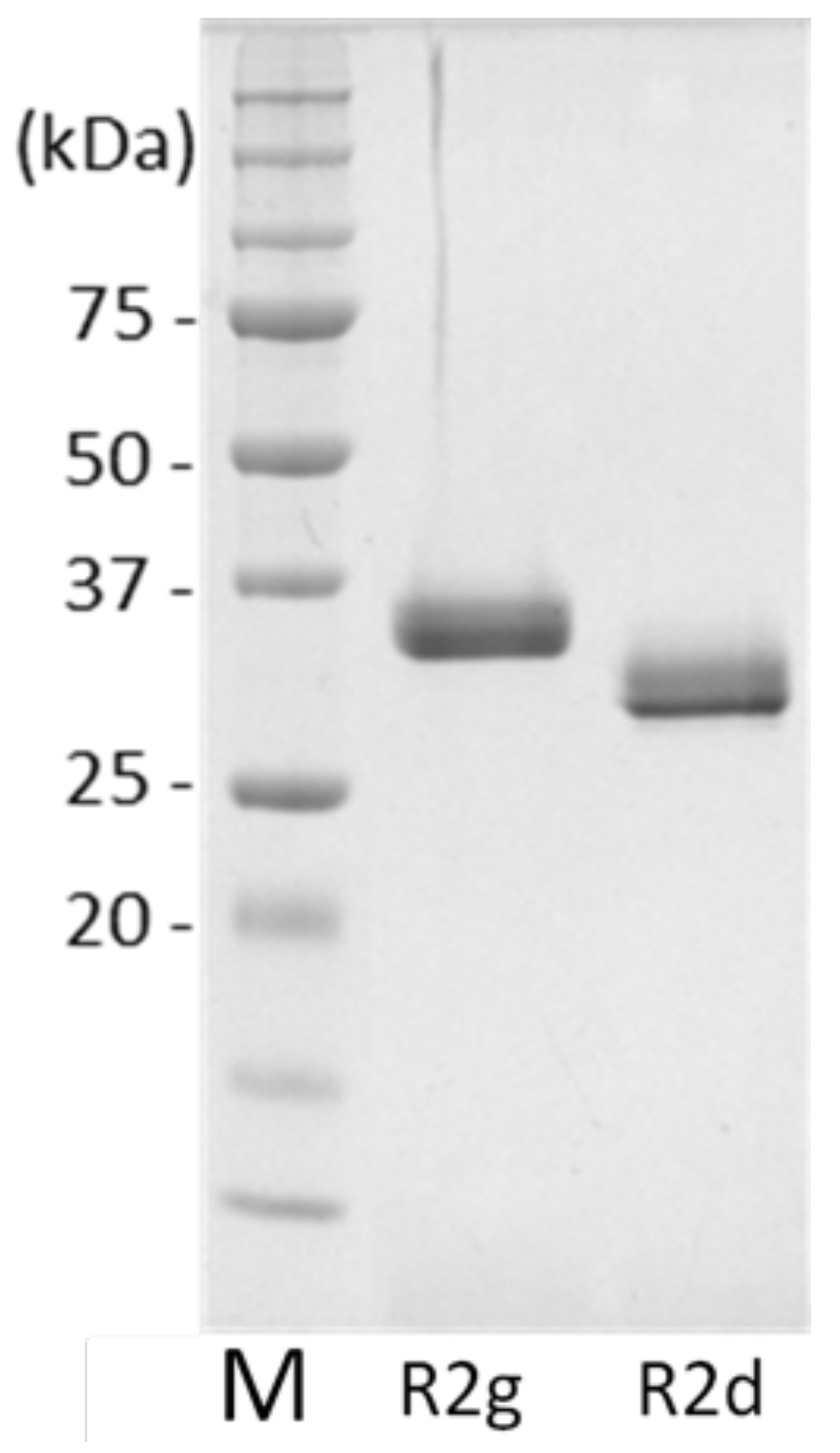


Figure S2. Normalized melting curves measured by thermal shift assay (TSA). Data showed that melting temperature of deglycosylated $\operatorname{IFNgR} 2\left(48^{\circ} \mathrm{C}\right)$ is slightly lower than of glycosylated variant $\left(50.5^{\circ} \mathrm{C}\right)$. The addition of $5 \mathrm{mM}$ TCEP to break the disulfide bonds lower the melting temperature just by one degree of Celsius of both deglycosylated and glycosylated IFNgR2 (47 and $49.5^{\circ} \mathrm{C}$, respectively).

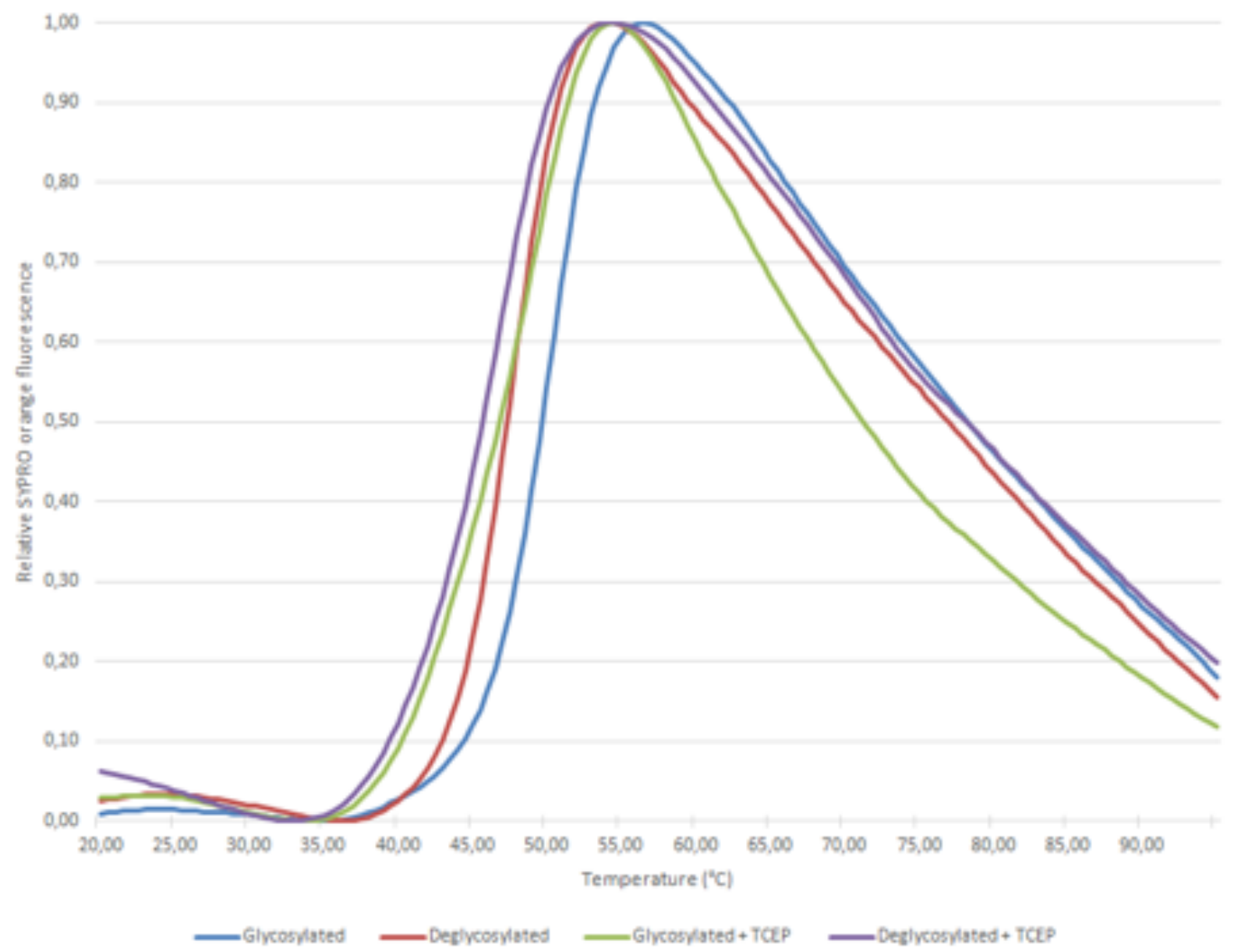


Figure S3. Residues responsible for stability of the IFNgR2 N-terminal (D1) domain. The color and thickness of the cartoon representation show relative interaction energy per residue ranging from low (blue) to high stabilizing values (red). The interaction energy within D1 was calculated at the DFT-D level (RI-TPSS/TZVP augmented with empirical dispersion term (Černý et al., 2007)) with the solvation effects described using COSMO in program TurboMole (Furche et al., 2014). All pairs of D1 residues within $4 \AA$ were extracted from the $5 \mathrm{eh} 1$ structure using VMD; it was 113 residues forming 580 pairs. Hydrogen atoms were added using OpenBabel (O'Boyle et al., 2011) and their positions were optimized at the DFTB-D level of theory as implemented in $\mathrm{dftb}+$ program (Rüger et al., 2015). Pairwise interaction energy was calculated at the DFT-D level and contributions involving each residue were summed.

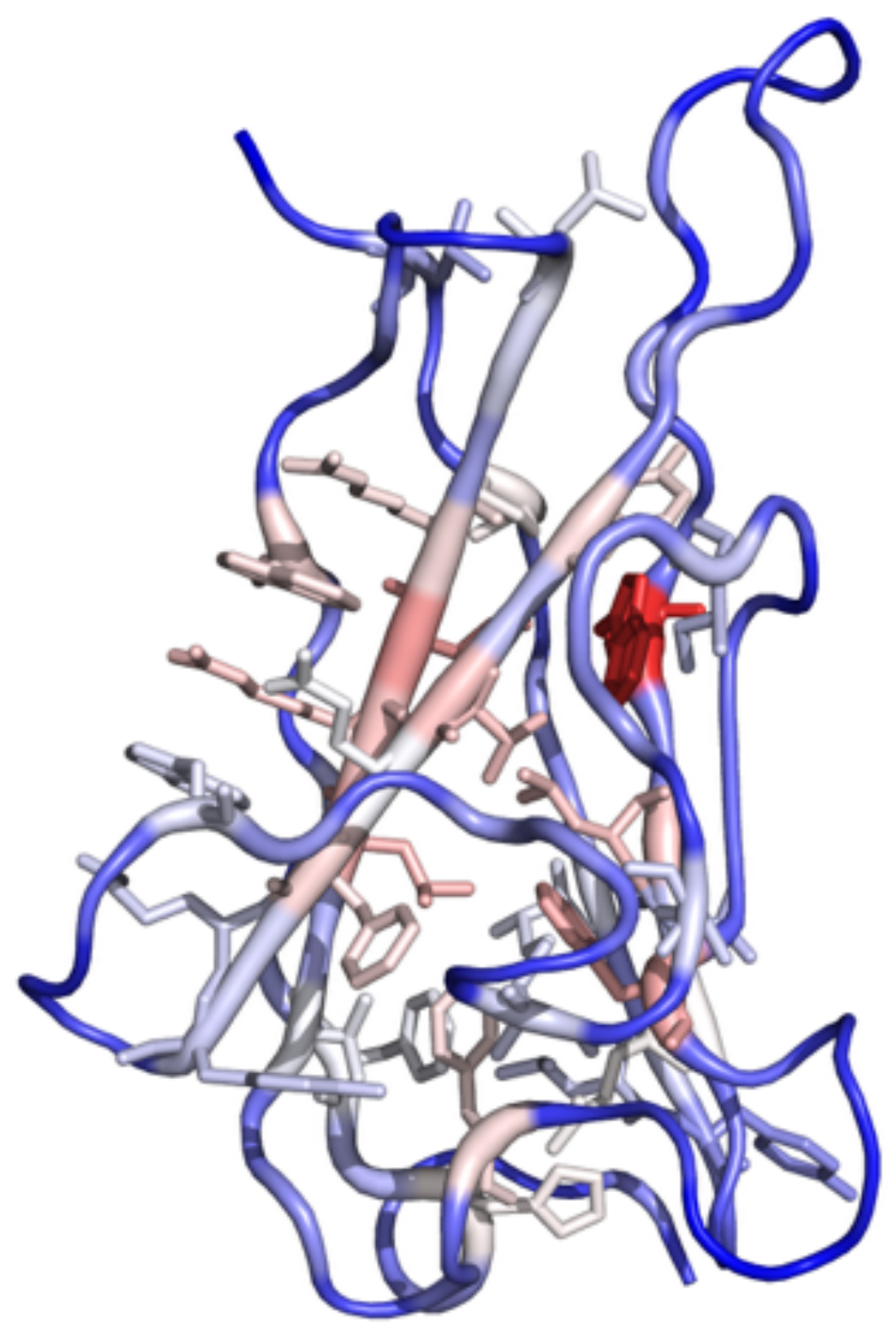




\section{Bibliography}

Cancino-Diaz, J. C., Reyes-Maldonado, E., Banuelos-Panuco, C. A., Jimenez-Zamudio, L., Garcia-Latorre, E., Leon-Dorantes, G., Blancas-Gonzalez, F., Paredes-Cabrera, G. \& Cancino-Diaz, M. E. (2002). J. Investig. Dermatol. 119, 1114-1120.

Černý, J., Jurečka, P., Hobza, P. \& Valdés, H. (2007). The Journal of Physical Chemistry A 111, 1146-1154.

Furche, F., Ahlrichs, R., Hättig, C., Klopper, W., Sierka, M. \& Weigend, F. (2014). Wiley Interdisciplinary Reviews: Computational Molecular Science 4, 91-100.

O'Boyle, N. M., Banck, M., James, C. A., Morley, C., Vandermeersch, T. \& Hutchison, G. R. (2011). Journal of Cheminformatics 3, 33-33.

Rüger, R., van Lenthe, E., Lu, Y., Frenzel, J., Heine, T. \& Visscher, L. (2015). Journal of Chemical Theory and Computation 11, 157-167. 